\title{
medicina intensiva
}

www.elsevier.es/medintensiva

\section{REVISIÓN}

\section{Hiperglucemia de estrés y su control con insulina en el paciente crítico: evidencia actual}

\author{
W. Manzanares* e I. Aramendi
}

Cátedra de Medicina Intensiva, Centro de Tratamiento Intensivo, Hospital de Clínicas, Facultad de Medicina-UDELAR, Montevideo, Uruguay

Recibido el 25 de agosto de 2009; aceptado el 6 de octubre de 2009

Disponible en Internet el 26 de noviembre de 2009

\section{PALABRAS CLAVE Hiperglucemia; Paciente crítico; Tratamiento intensivo con insulina; Hipoglucemia}

\section{KEYWORDS}

Hyperglycemia; Critical illness; Intensive insulin therapy;

Hypoglycemia

\begin{abstract}
Resumen
Objetivo: Analizar la evidencia actual sobre el control de la glucemia con insulina en el paciente crítico.

La hiperglucemia de estrés incrementa la morbimortalidad en el paciente crítico y se ha reconocido a la variabilidad de la glucemia como un predictor independiente de mortalidad. Inicialmente, los estudios Leuven han demostrado que el control estricto de la glucemia es capaz de reducir la mortalidad en pacientes críticos médicos y quirúrgicos. Sin embargo, esta estrategia terapéutica incrementa de modo significativo la incidencia de hipoglucemia grave. Recientemente, se han publicado los estudios Efficacy of Volume Substitution and Insulin Therapy in Severe Sepsis, GluControl y Normoglycemia in Intensive Care Evaluation and Survival Using Glucose Algorithm Regulation, los que han demostrado que el control estricto de la glucemia incrementa la mortalidad y la incidencia de hipoglucemia grave. Asimismo, un reciente metaanálisis indica que el control estricto de la glucemia podría ser beneficioso en pacientes críticos quirúrgicos. Futuras investigaciones deberían ser capaces de responder algunos interrogantes importantes surgidos a partir de los diferentes estudios existentes.
\end{abstract}

(c) 2009 Elsevier España, S.L. y SEMICYUC. Todos los derechos reservados.

Stress hyperglycemia and its control with insulin in critically ill patients. Current evidence

Abstract

Objective: To analyze the current evidence on glycemic control with insulin therapy in the critically ill.

Recent findings: Stress hyperglycemia in critically ill patients has been associated with increased morbidity and mortality. Furthermore, current evidence suggests that glucose variability has a predictive value for hospital mortality. Initially, the Leuven studies showed

*Autor para correspondencia.

Correo electrónico: wmanzanares@adinet.com.uy (W. Manzanares). 
that intensive insulin therapy was capable of reducing the mortality among surgical and medical ICU patients. Nevertheless, this strategy significantly increases the incidence of severe hypoglycemia.

Three important trials on glucose control have been published recently: the VISEP, the Glucontrol study and the NICE-SUGAR. They have shown that strict control of glycemia is associated with a higher incidence of mortality and severe hypoglycemia. Furthermore, according to a recent meta-analysis, intensive insulin therapy may be beneficial for patients admitted to a surgical ICU. Further studies should be able to address some queries about these results on glycemic control in the critically ill.

(c) 2009 Elsevier España, S.L. and SEMICYUC. All rights reserved.

\section{Introducción. Definición e importancia del tema}

La hiperglucemia de estrés, también denominada diabetes del estrés o diabetes por lesión aguda, es aquella hiperglucemia (nivel de glucosa sanguínea $>1,26 \mathrm{~g} / \mathrm{l}$ en ayuno o un valor superior a $2,0 \mathrm{~g} / \mathrm{l}$ medido en cualquier momento) que aparece en un paciente crítico u hospitalizado por enfermedad no crítica sin antecedentes previos de diabetes mellitus de tipo 1 o $2^{1}$. Esta forma de hiperglucemia es la consecuencia de una serie de alteraciones hormonales caracterizada por: a) incremento de las hormonas contrarreguladoras de la insulina (glucagón, cortisol, catecolaminas y hormona del crecimiento) y b) respuesta inflamatoria sistémica. Estos cambios son los causantes del incremento de la gluconeogenia y glucogenólisis hepática y de la resistencia periférica a la acción de la insulina que caracterizan el metabolismo glucídico durante el estrés ${ }^{1-5}$. De acuerdo con Sakarova et $\mathrm{al}^{2}$ la hiperglucemia de estrés es el resultado de la contraposición de efectos entre las hormonas contrarreguladoras de la insulina y la reserva funcional de las células $\beta$ del páncreas.

La hiperglucemia de estrés es un predictor pronóstico que actúa como un marcador de morbimortalidad durante la enfermedad crítica ${ }^{1,6-20}$. Existe evidencia creciente que asocia el grado de hiperglucemia y la duración de ésta al pronóstico de la enfermedad crítica ${ }^{6,12}$. Así, por ejemplo, en pacientes traumatizados, la hiperglucemia precoz se ha asociado a un número creciente de complicaciones infecciosas, aumento de la estancia en la $\mathrm{UCl}$ y de la estancia hospitalaria, así como a una mayor mortalidad ${ }^{17,18}$. Recientemente, en una serie retrospectiva $(n=555)$, Kreutziger et $\mathrm{al}^{9}$ han demostrado que la hiperglucemia al ingreso a la $\mathrm{UCl}(>135 \mathrm{mg} / \mathrm{dl})$ es un predictor independiente de mortalidad hospitalaria $(\mathrm{p}<0,0001)^{9}$.

Por su parte, entre los pacientes con infarto agudo de miocardio (IAM), la aparición de hiperglucemia se asocia a riesgo aumentado de insuficiencia cardíaca congestiva, shock cardiogénico y mortalidad hospitalaria ${ }^{20}$. Asimismo, en los pacientes con accidentes cerebrovasculares, la hiperglucemia permite predecir una pobre recuperación funcional y riesgo aumentado de muerte, en tanto que en el traumatismo craneoencefálico grave la hiperglucemia se ha asociado a mal control de la hemodinámica intracraneal, mal pronóstico funcional, mayor estancia hospitalaria y mayor mortalidad ${ }^{21,22}$.

\section{Metabolismo glucídico en la inflamación sistémica}

La hiperglucemia de estrés se define por la existencia de hiperglucemia, hiperinsulinemia con resistencia periférica a la acción de la insulina e hiperproducción de glucosa ${ }^{3}$. Esta hiperglucemia del estrés es secundaria a: a) incremento de la gluconeogenia hepática por aumento de las hormonas contrarreguladoras de la insulina (glucagón, cortisol, catecolaminas y hormona del crecimiento) y, en particular, al aumento de la relación glucagón/insulina a nivel portal; b) resistencia periférica a la acción de la insulina por inhibición del transportador de glucosa dependiente de la insulina transportador de glucosa 4 (GLUT-4) en el tejido adiposo, músculo estriado esquelético y cardíaco $0^{5,17}$. El grado de la hiperglucemia de estrés guarda una correlación positiva con la gravedad de la respuesta inflamatoria sistémica ${ }^{3}$. Esto se debe a que ésta es secundaria a una respuesta neuroendocrina con hipersecreción de las hormonas contrarreguladoras de la insulina y a la liberación de citoquinas proinflamatorias, factor de necrosis tumoral $\alpha$ (FNT- $\alpha$ ) y las interleucinas (IL) 1 y $6^{3,23,24}$. Esta respuesta neuroendocrina es causante del aumento de la glucogenólisis y de la gluconeogenia hepática ${ }^{1-4}$. Ambas catecolaminas (adrenalina y noradrenalina) son causantes directas del incremento de la glucogenólisis hepática, en tanto que la adrenalina explica la respuesta glucogenolítica en el músculo estriado esquelético ${ }^{3}$. Esta respuesta glucogenolítica es de corta duración y se agota en un período de tiempo no mayor a las $36 \mathrm{~h}$.

Por su parte, la gluconeogenia (síntesis de glucosa a partir de sustratos no glucídicos, piruvato, alanina, lactato, glutamina y glicerol) es uno de los mayores determinantes de la hiperglucemia de estrés ${ }^{3}$. En condiciones fisiológicas, ante el aumento de la gluconeogenia se produce un incremento en la secreción de insulina con inhibición de la secreción pancreática de glucagón ${ }^{3,17}$. Sin embargo, en los estados de inflamación sistémica las catecolaminas endógenas o exógenas son capaces de interferir con esta respuesta de retroalimentación negativa, lo que favorece la persistencia de la hiperglucemia. Los sustratos neoglucogénicos más importantes son los siguientes: a) piruvato; b) lactato (secundario al incremento del flujo glucolítico y a la inhibición del complejo piruvato deshidrogenasa por acción de las citoquinas proinflamatorias de IL-1 y de FNT- $\alpha$ ); c) alanina, derivada del catabolismo muscular, la que es 
convertida en glucosa vía el ciclo de Cori, y d) glicerol, producto final de la lipolisis, explica hasta el $20 \%$ de la producción hepática de glucosa ${ }^{3}$.

En los estados de inflamación sistémica, la captación total de glucosa está aumentada y es más ostensible en el sistema nervioso central y periférico y en las células sanguíneas ${ }^{3,17}$.

Por otra parte, la resistencia hepática a la acción de la insulina se caracteriza por un aumento de los niveles plasmáticos de la proteína ligadora del insulin like growth factor binding-1; estudios recientes permiten afirmar que este incremento guarda una relación directa con el riesgo de mortalidad $^{17}$.

\section{Efectos tóxicos secundarios de la hiperglucemia}

La hiperglucemia es un trastorno metabólico que se caracteriza por ser potencialmente más tóxico en el paciente crítico con relación a los pacientes con diabetes mellitus previa. La hiperglucemia per se es capaz de inducir un estado proinflamatorio. Evidencia creciente permite afirmar que las razones causantes de la toxicidad atribuible a la hiperglucemia son la excesiva sobrecarga celular de glucosa y el estrés oxidativo celular ${ }^{17,18}$.

a) Excesiva sobrecarga celular de glucosa. La glucosa es transportada hacia el interior celular independientemente de la insulina en hepatocitos, inmunocitos, epitelios y tejido nervioso, a través de los transportadores: GLUT-1, GLUT-2 (hepatocitos, epitelio de la mucosa del tracto intestinal, células $\beta$ de los acinos pancreáticos y epitelio tubular renal) y GLUT-3 (neuronas). En los estados de inflamación sistémica se asiste a una sobreestimulación de los transportadores GLUT-1 y GLUT-3, fenómeno que afecta particularmente a los endotelios, las neuronas, la astroglía y el músculo liso ${ }^{18}$. De acuerdo con los conocimientos actuales, las citoquinas causantes de la resistencia a la insulina son la IL-1, IL-6 y el FNT- $\alpha$, así como la proteína $C$ reactiva ${ }^{3,23,24}$. Por otra parte, el músculo esquelético y cardíaco que captan la glucosa por un mecanismo dependiente de la insulina mediante el transportador GLUT-4 se encuentran protegidos de la sobrecarga de glucosa ${ }^{17}$.

b) Estrés oxidativo celular. Se debe a la generación de radicales libres $(\mathrm{RL})$ por excesiva glucólisis y fosforilación oxidativa. La sobrecarga citosólica de glucosa conlleva a un incremento de la generación de piruvato y RL, en particular superóxido y peroxinitrito, que son la causa de estrés oxidativo, nitrativo y de apoptosis celular, eventos éstos que son consecuencia directa de la hiperglucemia de estrés ${ }^{17}$. Van den Berghe et $\mathrm{al}^{17}$ han demostrado que el tratamiento intensivo con insulina evita la aparición o promueve la reversión de los cambios estructurales a nivel mitocondrial en los hepatocitos; asimismo, estos cambios celulares se han correlacionado con alteraciones funcionales, tales como una mayor actividad de los complejos I y iv de la cadena respiratoria mitocondrial ${ }^{3,5,17}$.

En la célula la sobrecarga de glucosa promueve la activación y traslocación nuclear del factor de transcripción nuclear $\kappa-B$ con la consiguiente expresión de genes proinflamatorios (IL-1, IL-2, IL-6, IL-8, FNT- $\alpha$ y óxido nítrico [NO] sintasa inducible) ${ }^{3}$. Por otra parte, la hiperglucemia es causa de disfunción inmunitaria. En tal sentido, la hiperglucemia afecta la inmunidad celular y provoca: a) reducción de la activación de neutrófilos; b) disminución del quimiotactismo, y c) disminución de la fagocitosis, de la actividad bactericida intracelular e hiperproducción de $\mathrm{RL}^{3,4}$. Finalmente, los efectos de la hiperglucemia sobre la inmunidad humoral incluyen la inactivación de las inmunoglobulinas por glucosilación ${ }^{3}$ y el estímulo de la síntesis de IL-6 y FNT- $\alpha$ por los monocitos periféricos.

\section{Efectos metabólicos protectivos de la insulina}

La infusión de insulina es capaz de revertir la resistencia periférica a ésta en el músculo esquelético mediante el aumento de la captación celular de glucosa actuando sobre el ARNm del transportador GLUT-4 y de la enzima hexoquinasa ${ }^{3,17}$.

La insulina ejerce sus efectos protectivos mediante 2 mecanismos diferentes: a) directos (vinculados a sus propiedades antiinflamatorias por acción directa sobre el factor de transcripción nuclear $\kappa-B)^{3,17}$ y b) indirectos, mediante el control de la glucemia ${ }^{18,25,26}$.

Sobre el metabolismo lipídico, Messoten et $\mathrm{al}^{27}$ han demostrado que la insulina es capaz de controlar la hipertrigliceridemia ( $p<0,0001)$, así como también es capaz de reducir los niveles de ácidos grasos libres e incrementar los valores de colesterol-HDL $(p=0,005)$ y colesterol-LDL $(p=0,007)^{27}$. Por su parte, el análisis de regresión logística multivariada, realizado por el mismo grupo, demostró que el control de los lípidos plasmáticos antes que el control de la glucemia fue causante de la disminución significativa de la mortalidad y del desarrollo de disfunción orgánica múltiple ${ }^{27}$.

A nivel del metabolismo proteico la insulina exhibe sus propiedades anabólicas, atenúa el hipercatabolismo y promueve la síntesis proteica en el músculo esqueléti$\mathrm{CO}^{1,3,17}$.

Por último, la insulina ayuda a revertir la disfunción endotelial en el choque séptico. En efecto, la infusión de insulina es capaz de inhibir la producción de NO vía inhibición de la enzima NO sintasa inducible y mediante la inhibición indirecta de la enzima constitutiva NO sintasa endotelial $y$, por consiguiente, de la síntesis de NO endotelial $^{24,27}$.

\section{Evidencia actual sobre control de la glucemia en el paciente crítico: tratamiento intensivo frente al tratamiento convencional}

El tratamiento de la hiperglucemia de estrés ha sido uno de los aspectos más estudiados y controvertidos en medicina intensiva en los últimos años. Diferentes estrategias se han desarrollado para lograr el control de la glucemia en el paciente crítico (infusión intravenosa de insulina en función del perfil glucémico, administración de bolos de insulina subcutánea en pacientes con hemodinamia estable y combinación de estas estrategias). 
Sin embargo, a partir del primer estudio Leuven ${ }^{11}$ la normoglucemia (independientemente del valor considerado como tal) en el paciente crítico se ha obtenido mediante la infusión intravenosa de insulina, excepto en algunos ejemplos de pacientes críticos crónicos donde estrategias menos agresivas deben emplearse.

Van den Berghe et $\mathrm{al}^{11}$ en 1.548 pacientes quirúrgicos críticos compararon el control estricto de la glucemia $(0,80-1,10 \mathrm{~g} / \mathrm{l})$ frente a un objetivo convencional $(1,80-2,00 \mathrm{~g} / \mathrm{l})$ y demostraron que el control estricto se asociaba a una reducción de la mortalidad en la $\mathrm{UCl}$ del $43 \%$ (el 8,0 al 4,6\%; $p<0,04$ ), este beneficio era más notable en los pacientes que requerían estancia en la $\mathrm{UCI}$ superior a 5 días en los que la reducción de la mortalidad fue del 20,2 al $10,6 \%(p=0,005)^{9}$. Por su parte, los autores demostraron una reducción de la mortalidad hospitalaria del 10,9 al 7,2\% $(p=0,01)$ y del 26,3 al $16,8 \%(p=0,01)$ en los pacientes con estancia prolongada en la $\mathrm{UCl}$ a los que se les realizó control estricto ${ }^{11}$.

Asimismo, el tratamiento intensivo con insulina se asoció a una reducción del $46 \%$ en la incidencia de bacteriemia, el $41 \%$ de insuficiencia renal aguda (IRA) que requiería hemofiltración, el $44 \%$ de polineuropatía del paciente crítico $y$ el $50 \%$ de transfusión de glóbulos rojos (tabla 1$)^{11}$. Sin embargo, el estudio Leuven 1 ha recibido importantes críticas. Así, por ejemplo, la administración de una importante carga de glucosa parenteral (8-12 g/h, 200-300 g/día) el día uno de ingreso a la $\mathrm{UCl}$ es una práctica inhabitual, que no está indicada y que per se es capaz de agravar la hiperglucemia. Por su parte, la incidencia de hipoglucemia grave fue 5 veces mayor en el grupo de tratamiento intensivo (5,2 vs. 0,85\%) $\mathrm{y}$, en particular, en pacientes que no sobrevivieron a la enfermedad crítica lo cual significó una crítica mayor a la estrategia de control estricto de la glucemia ${ }^{11}$. Finalmente, el 63\% de los participantes en cada grupo eran pacientes de cirugía cardíaca, en tanto que la puntuación APACHE II al ingreso fue baja (mediana: 9 , rango entre 7 y 13) ${ }^{11}$.

Posteriormente, Krinsley et $\mathrm{al}^{12}$ evaluaron los efectos del control estricto de la glucemia (valor promedio de glucemia de $132 \mathrm{mg} / \mathrm{dl}$ ) en pacientes críticos médicos y quirúrgicos. La implementación del protocolo de control estricto redujo en un $29,3 \%$ la mortalidad hospitalaria, un 10,8\% la estancia en la $\mathrm{UCI}$, un $75 \%$ la IRA y en un $18,7 \%$ los requerimientos de transfusión de glóbulos rojos ${ }^{12}$.

El estudio Leuven $2^{19}$ publicado en el año 2006 evaluó los efectos del control estricto de la glucemia en pacientes críticos médicos mediante la aplicación del protocolo del estudio Leuven 1. El análisis por intención de tratar de los

Tabla 1 Estudios más importantes publicados sobre control de la glucemia y tratamiento intensivo con insulina en pacientes críticos

\begin{tabular}{|c|c|c|c|c|}
\hline Estudio & $\begin{array}{l}\text { Número de } \\
\text { pacientes }\end{array}$ & $\begin{array}{l}\text { Población de } \\
\text { estudio }\end{array}$ & $\begin{array}{l}\text { Nivel de glucemia } \\
\text { (mg/dl) }\end{array}$ & Resultados \\
\hline $\begin{array}{l}\text { Van den Berghe et } \\
\text { al, } 2001^{11}\end{array}$ & 1.548 & Quirúrgicos & $153-103$ & $\begin{array}{l}\downarrow \text { mortalidad } \\
\downarrow \text { morbilidad } \\
\downarrow \text { IRA } \\
\downarrow \text { polineuropatía del paciente } \\
\text { crítico } \\
\downarrow \text { necesidad de VM } \\
\downarrow \text { requerimiento de transfusiones } \\
\downarrow \text { costos }\end{array}$ \\
\hline $\begin{array}{l}\text { Krinsley et al, } \\
2006^{12}\end{array}$ & 1.600 & Médico-quirúrgicos & $152-131$ & $\begin{array}{l}\downarrow \text { mortalidad } \\
\downarrow \text { IRA } \\
\downarrow \text { estadía en la UCI }\end{array}$ \\
\hline $\begin{array}{l}\text { Van den Berghe et } \\
\text { al, } 2006^{19}\end{array}$ & 1.200 & Médicos & $160-105$ & $\begin{array}{l}\downarrow \text { mortalidad } \\
\downarrow \text { morbilidad } \\
\downarrow \text { IRA } \\
\downarrow \text { polineuropatía del paciente } \\
\text { crítico } \\
\downarrow \text { necesidad de VM }\end{array}$ \\
\hline Reed et al, $2003^{29}$ & 7.261 & Quirúrgicos & $141-129$ & $\begin{array}{l}\downarrow \text { abscesos abdominales } \\
\downarrow \text { días de VM }\end{array}$ \\
\hline $\begin{array}{l}\text { NICE-SUGAR, } \\
2009^{30}\end{array}$ & 6.100 & Médico-quirúrgicos & $140-108$ & $\begin{array}{l}\uparrow \text { riesgo de hipoglucemia } \\
\uparrow \text { mortalidad }\end{array}$ \\
\hline $\begin{array}{l}\text { Brunkhorst et al, } \\
2008^{31}\end{array}$ & 488 & Sepsis/shock séptico & $151-112$ & $\begin{array}{l}\uparrow \text { riesgo de hipoglucemia } \\
\uparrow \text { mortalidad } \\
\text { Suspendido precozmente }\end{array}$ \\
\hline Devos et al, $2008^{32}$ & 855 & Médico-quirúrgicos & $\begin{array}{l}147(127-163)-118 \\
(109-131)\end{array}$ & $\begin{array}{l}\uparrow \text { riesgo de hipoglucemia } \\
\text { Suspendido precozmente }\end{array}$ \\
\hline Arabi et al $2008^{33}$ & 523 & Médico-quirúrgicos & 115 vs. 171 & $\begin{array}{l}\uparrow \text { hipoglucemia } \\
\text { Sin diferencias en la mortalidad }\end{array}$ \\
\hline
\end{tabular}

IRA: insuficiencia renal aguda; NICE-SUGAR: Normoglycemia in Intensive Care Evaluation and Survival Using Glucose Algorithm Regulation; UCI: unidad de cuidados intensivos; VM: ventilación mecánica. 
1.200 pacientes enrolados no demostró una reducción significativa de la mortalidad hospitalaria (40 vs. $37,3 \%$ $[\mathrm{p}=0,33]$ en los grupos control y terapéutico, respectivamente) ${ }^{19}$. Sin embargo, la incidencia de nueva IRA se redujo del 8,9 al 5,9\%; $p=0,04)$, así como también se redujeron los tiempos de ventilación mecánica $(p=0,03)$ y la estancia en la $\mathrm{UCI}(\mathrm{p}=0,04)^{19}$. Este estudio demostró una reducción significativa de la mortalidad entre aquellos pacientes que estaban durante más de 3 días en la $\mathrm{UCl}$ (52,5 vs. 43,0\%; $\mathrm{p}=0,009)$; por su parte, en este grupo de estancia prolongada en la UCI también se apreció una reducción de la incidencia de nueva IRA $(12,6$ vs. $8,3 \% ; p=0,05)$, duración de la ventilación mecánica $(p<0,001)$, días de $\mathrm{UCl}(\mathrm{p}=0,02)$ y estancia hospitalaria $(p<0,001)^{19}$.

En el año 2006 Van den Berghe et $\mathrm{al}^{28}$ publicaron los resultados del análisis por intención de tratar en la población mixta de pacientes críticos y quirúrgicos de los 2 estudios Leuven ${ }^{11,19}$. Los resultados de este análisis revelan que el tratamiento intensivo con insulina reduce la morbimortalidad de la enfermedad crítica en especial cuando el tratamiento se mantiene por un período de tiempo no menor a 3 días $^{28}$. Asimismo, este análisis concluyó que dichos beneficios eran independientes de la carga de glucosa parenteral y que dicha terapéutica no era nociva en aquellos pacientes tratados durante menos de 3 días $^{28}$. Por su parte, todos los grupos de pacientes críticos exhibieron los beneficios del tratamiento intensivo con excepción de aquellos pacientes previamente diabéticos ${ }^{28}$.

Reed et $\mathrm{al}^{29}$ han evaluado la aplicación de un protocolo de tratamiento intensivo con insulina entre los años 2003-2006 en una $\mathrm{UCl}$ de trauma $(\mathrm{n}=3.536)$. En dicha serie, los autores demostraron que la aplicación del protocolo fue capaz de reducir temporariamente la mortalidad $(p<0,01)$, así como la incidencia de abscesos abdominales $(p=0,002)$ y los días de ventilación mecánica $(\mathrm{p}=0,03)^{29}$.

Sin embargo, evidencia reciente se contrapone a lo anteriormente establecido. En el año 2009 se han publicado los resultados del estudio multicéntrico Normoglycemia in Intensive Care Evaluation and Survival Using Glucose Algorithm Regulation (NICE-SUGAR) ${ }^{30}$, hasta el momento actual el estudio más importante sobre el control de la glucemia en pacientes críticos realizado por la Australia and New Zealand Intensive Care Society y por los Canadian Critical Care Groups. Este estudio enroló 6.104 pacientes con el objetivo de evaluar el impacto de 2 rangos de glucemia $(0,80-1,10 \mathrm{~g} / \mathrm{l}$ y 1,40 $1,80 \mathrm{~g} / \mathrm{l})$ sobre la mortalidad a los 90 días. El análisis de los resultados revela que no existieron diferencias entre ambos grupos en cuanto a estancia en la $\mathrm{UCl}(\mathrm{p}=0,84)$, estancia hospitalaria $(p=0,86)$, días de ventilación mecánica $(p=0,56)$ y uso de técnicas de reemplazo renal $(p=0,39)^{30}$. Por su parte, la incidencia de hipoglucemia grave (glucemia $\leq 0,40 \mathrm{~g} / \mathrm{l}$ ) se constató en 206/3.016 pacientes $(6,8 \%)$ del grupo de tratamiento intensivo frente a 15/3.014 pacientes $(0,5 \%)$ en el grupo de tratamiento convencional $(p<0,001)^{30}$. Finalmente, la mortalidad en el grupo de tratamiento intensivo fue significativamente mayor que en el grupo de tratamiento convencional $(27,5$ vs. $24,9 \%$; $p=0,02)$. Asimismo, no hubo diferencias en la mortalidad entre los pacientes críticos quirúrgicos y médicos (OR en los grupos intensivo y convencional fue de 1,31 y de 1,07 , respectivamente; $p=0,1)$. Los resultados del NICE-SUGAR permiten concluir que un objetivo convencional de glucemia $(<1,80 \mathrm{~g} / \mathrm{l})$ se asocia a una menor mortalidad que un objetivo intensivo ${ }^{30}$.

El estudio multicéntrico alemán Efficacy of Volume Substitution and Insulin Therapy in Severe Sepsis ${ }^{31}$ realizado por el grupo SepNet aleatorizó pacientes para mantener normoglucemia con tratamiento intensivo o tratamiento convencional con insulina asociado a Pentastarch (hidroxietil almidón) al $10 \%$. Este estudio se interrumpió precozmente luego de haber reclutado 537 pacientes debido a la idéntica mortalidad a los 28 días en ambos grupos y a la mayor incidencia de hipoglucemia grave en el grupo de control estricto $(17,0$ vs. $4,1 \% ; \mathrm{p}<0,001)$ y de episodios adversos graves vinculados a hipoglucemia $(10,9 \text { vs. } 5,2 \% ; p=0,01)^{31}$.

Por su parte, el estudio multicéntrico europeo GluControl $^{32}$ se interrumpió precozmente debido a la inaceptablemente elevada incidencia de hipoglucemia en el grupo de tratamiento intensivo $(8,7$ vs. 2,$7 ; p<0,0001)$ y una mortalidad semejante $(15,3 \text { vs. } 17,2 \%)^{32}$. Arabi et $a^{33}$ aleatorizaron y asignaron 523 pacientes críticos a un objetivo de tratamiento intensivo $(0,80-1,10 \mathrm{~g} / \mathrm{l})$ o convencional $(1,80-2,00 \mathrm{~g} / \mathrm{l})$. Los resultados demuestran que no existen diferencias entre ambos grupos en la mortalidad $(p=0,30)$, aunque la incidencia de hipoglucemia fue significativamente mayor en el grupo de tratamiento intensivo (28,6 vs. $3,1 \% ; \mathrm{p}<0,0001$ o $6,8 / 100$ días de tratamiento vs. $0,4 / 100 ; p<0,0001)^{33}$.

Las últimas recomendaciones de la Surviving Sepsis Campaign $2008^{34}$ recomendaron mantener un nivel de glucemia por debajo de $1,50 \mathrm{~g} / \mathrm{l}$ (recomendación grado $2 \mathrm{C}$ ). Asimismo, las referidas guías recomiendan administrar glucosa parenteral mientras se infunde insulina, se monitoriza con glucemias seriadas cada 1 o $2 \mathrm{~h}$ hasta la estabilización del perfil glucémico y se continúa luego con controles seriados cada $4 \mathrm{~h}$ (recomendación grado $1 \mathrm{C})^{34}$.

Krinsley et $\mathrm{al}^{35}$ han indicado una estrategia stepwise para controlar la glucemia, la que se ha denominado "control efectivo y seguro de la glucemia" (safe, effective glucose control $)^{35}$. Esta estrategia persigue como objetivo el control de la incidencia de hiperglucemia y reduce los efectos adversos de la hipoglucemia. Para lograr dicho objetivo se indica como máximo nivel de glucemia $1,50 \mathrm{~g} / \mathrm{l}$; este objetivo está basado en estudios retrospectivos que describen un incremento de la mortalidad con niveles de glucemia superiores a dicha cifra ${ }^{35}$.

Un metaanálisis recientemente publicado ${ }^{36}$, que incluye los resultados del estudio NICE-SUGAR ha concluido que en una $\mathrm{UCl}$ médica el tratamiento intensivo con insulina no es capaz de disminuir la mortalidad (RR: 1,00; IC 95\%: $0,78-1,28)$, en tanto que en una $\mathrm{UCl}$ quirúrgica la referida estrategia podría ser de utilidad (RR: 0,63; IC 95\%: $0,44-0,91)^{36}$. El análisis de los resultados de este metaanálisis permite concluir que el tratamiento intensivo con insulina no parece tener impacto positivo sobre la mortalidad del paciente crítico e incrementa de modo significativo la incidencia de hipoglucemia ${ }^{36}$.

Las actuales recomendaciones de la American Diabetes Association y de la American Association of Endocrinologists $^{37}$ se basan en los siguientes puntos para el control de la glucemia en el paciente crítico:

1. iniciar la infusión de insulina cuando la glucemia sea $>1,80 \mathrm{~g} / \mathrm{l}$; 
2. el nivel óptimo de glucemia debe ser de 1,40-1,80 g/l;

3. la insulina intravenosa en perfusión continua es el método de elección para controlar la glucemia;

4. es necesaria la realización e implementación de protocolos de control de la glucemia en cada $\mathrm{UCl}$,

5. y, la monitorización de la glucemia es esencial para minimizar el riesgo de hipoglucemia y optimizar el perfil glucémico.

Así pues, de acuerdo con la evidencia actual el rango óptimo de glucemia en pacientes críticos debería ser de 1,40$1,80 \mathrm{~g} / \mathrm{l}^{37}$.

\section{Variabilidad de la glucemia: predictor independiente de mortalidad en la enfermedad crítica}

La variabilidad de la glucosa (VG) es un factor de riesgo emergente y constituye un predictor independiente de mortalidad hospitalaria en pacientes críticos y, en particular, en aquéllos con sepsis grave/shock séptico ${ }^{38-40}$. Esta VG refleja las fluctuaciones de la glucemia durante la enfermedad crítica y se considera actualmente un factor de relevancia semejante al mantenimiento de la glucemia dentro de un rango normal o "próximo a lo normal". La serie retrospectiva de Krinsley ${ }^{40}$ ha demostrado que el efecto deletéreo de la VG es más ostensible en aquellos pacientes previamente no diabéticos y en los que mantienen niveles medios de glucemia dentro del rango de euglucemia

(70-99 mg/dl) donde la mortalidad pasa del 5,9 al 30,1\% (entre los cuartiles 1 y 4$)^{40}$. Por otra parte, la VG incrementa el riesgo de hipoglucemia grave $(<40 \mathrm{mg} / \mathrm{dl})$, la que ha demostrado ser un predictor de mortalidad en el paciente crítico $^{41,42}$.

\section{Estrategias de control de la glucemia en grupos específicos de pacientes críticos}

\section{Infarto agudo de miocardio}

Las infusiones de glucosa-insulina-potasio en el curso de un IAM con la finalidad de controlar la glucemia han demostrado tener un impacto positivo sobre su pronóstico ${ }^{20}$; sin embargo, los resultados existentes son controvertidos.

En el estudio Diabetes Insuline-Glucose in Acute Myocardium Infarction ${ }^{43}$ se incluyeron 620 pacientes para comparar la insulina frente al placebo con la finalidad de reducir la glucemia por debajo de $200 \mathrm{mg} / \mathrm{dl}$ en el período post-IAM. El estudio reveló que la insulinoterapia redujo la mortalidad en un porcentaje superior al $50 \%$, este hecho fue más ostensible en los pacientes diabéticos que previamente al episodio coronario no requerían insulina. Sin embargo, el estudio Diabetes Insuline-Glucose in Acute Myocardium Infarction$2^{44}$ no fue capaz de confirmar estos hallazgos, aunque el análisis post hoc permitió concluir que la hiperglucemia era un predictor independiente de mortalidad en el IAM. Este hecho permitió inferir que el control de la glucemia en la etapa aguda del IAM mejora el pronóstico a largo plazo.

Por su parte, el estudio CREATE-ECLA ${ }^{45}$ no demostró resultados beneficiosos con la infusión de glucosa-insulina- potasio en el curso de un IAM con supradesnivel del segmento ST.

\section{Postoperatorio de cirugía cardíaca}

Existe amplia evidencia que avala el control de la glucemia en el postoperatorio de cirugía cardíaca $(\mathrm{POCC})^{46,47}$. En tal sentido, en el estudio Leuven $1^{11}$ el $63 \%$ de los pacientes en el grupo de tratamiento convencional y el $62 \%$ en el grupo de tratamiento intensivo eran POCC. Por su parte, Gandhi et $\mathrm{al}^{48}$ aleatorizaron 2 grupos de pacientes, los que recibieron insulina durante la cirugía para mantener niveles de glucemia entre $80-110 \mathrm{mg} / \mathrm{dl}(\mathrm{n}=199)$ y un grupo de tratamiento convencional $(n=201)$, los que recibieron insulina cuando la glucemia fue mayor a $200 \mathrm{mg} / \mathrm{dl}$. Ambos grupos recibieron insulina para mantener la normoglucemia durante el POCC. El análisis de los resultados demuestra una mayor incidencia de accidente cerebrovascular (ACV) isquémico ( 8 vs. $1 ; p=0,02)$ y mortalidad $(p=0,061)$ en el grupo de tratamiento intensivo ${ }^{48}$. Finalmente, el control de la glucemia con insulina intravenosa en los primeros 3 días del POCC se ha asociado a una menor incidencia de mediastinitis anterior $(60 \%)$ e infección de la esternotomía (2,4 a 1,5\%), así como con un descenso significativo de la mortalidad ${ }^{49,50}$.

\section{Enfermedad neurológica aguda grave}

En la enfermedad neurológica grave traumática o vascular, la hiperglucemia es un predictor independiente de resultados negativos, por lo que el control de la glucemia se recomienda ampliamente en la lesión encefálica estructural o metabólica ${ }^{21,22,51,52}$. En el ACV isquémico la hiperglucemia se ha asociado a un incremento de la morbimortalidad; en tal sentido, estudios de experimentación animal han mostrado una expansión en el tamaño del área de isquemia y de penumbra isquémica en presencia de hiperglucemia ${ }^{51,52}$. Baird et $\mathrm{al}^{52}$ evaluaron el perfil glucémico mediante monitorización continua de la glucemia plasmática y capilar en 25 pacientes con ACV isquémico. El análisis de regresión múltiple demostró que la hiperglucemia (evaluada por ambos métodos) estuvo independientemente asociada a un mayor tamaño del área de infarto y a peor resultado funcional ${ }^{52}$.

Por su parte, en la hemorragia subaracnoidea, Frontera et $\mathrm{al}^{53}$ demostraron que la hiperglucemia se asocia a una estancia más prolongada en la $\mathrm{UCl}$, mayor número de complicaciones neurológicas (infarto o hidrocefalia) y complicaciones extraneurológicas (infecciosas o insuficiencia cardíaca), así como a riesgo de muerte o deterioro funcional ${ }^{53}$.

En el año 2007 Bilotta et $\mathrm{al}^{54}$ en 78 pacientes con hemorragia subaracnoidea constataron una reducción en la incidencia de infecciones en el grupo de pacientes a los que se les realizó tratamiento intensivo con insulina, en tanto que no existieron diferencias significativas en la incidencia de complicaciones neurológicas, en particular, vasoespasmo $(\mathrm{p}=0,7)$ y resultados globales a los 6 meses (mortalidad: 15 vs. $18 \% ; p=0,9)^{54}$.

En el año 2005 Van den Berghe et $\mathrm{al}^{15}$ publicaron los resultados del análisis de los 63 pacientes con lesión encefálica aislada extraídos de los 1.548 pacientes del estudio Leuven $1^{11}$. En dicha serie los pacientes a los que se 
les realizó control estricto de la glucemia presentaron un mejor control de la hemodinámica cerebral con valores óptimos de presión intracraneana, menores requerimientos de fármacos vasopresores, así como una menor incidencia de convulsiones y de diabetes insípida central ${ }^{15}$. Finalmente, los pacientes a los que se les realizó tratamiento intensivo con insulina presentaban menor incidencia de polineuropatía del paciente crítico y mejor recuperación funcional evaluada por score de Karnofsky al año del episodio neurológico ${ }^{15}$.

Sin embargo, Vespa et $\mathrm{al}^{55}$ en un estudio retrospectivo compararon 30 controles históricos a los que se les realizó control convencional frente a 14 pacientes a los que se les realizó control estricto de la glucemia. Los autores observaron en este grupo signos de sufrimiento energético cerebral traducido en un incremento de los niveles de glutamato y de la relación lactato/piruvato y una reducción en los niveles de glucosa en la microdiálisis cerebral. Por otra parte, la estrategia de tratamiento intensivo no estuvo asociada a una mejoría de resultados clínicos ni de la mortalidad (14 vs. $15 \% ; p=0,9)^{55}$.

En la actualidad existe suficiente evidencia que permite afirmar que el tratamiento intensivo con insulina en pacientes con enfermedad neurológica aguda grave conduce a neuroglucopenia relativa con disfunción energética neuronal, que es causa de lesión encefálica secundaria, en particular cuando el nivel de glucemia sistémica es inferior a $0,80 \mathrm{~g} / \mathrm{l}^{22}$. Así pues, de acuerdo con estudios clínicos y de experimentación animal es posible afirmar que el control estricto de la glucemia en el paciente neurocrítico es deletéreo, por lo que el rango de glucemia óptima en esta categoría de pacientes críticos debe ser de 1,10-1,80 g/ $\mathrm{l}^{22}$.

\section{Hipoglucemia como complicación del control de la glucemia en pacientes críticos}

Existen diferentes valores de corte para definir hipoglucemia, los más comúnmente utilizados son $40 \mathrm{mg} / \mathrm{dl}$ o $45 \mathrm{mg}$ / $\mathrm{dl}^{56-59}$. La incidencia de hipoglucemia descrita en los diferentes estudios es ampliamente variable y esto obedece a la definición utilizada y al valor objetivo de glucosa definido en el protocolo de estudio. En tal sentido, cuando la hipoglucemia se define por un valor inferior a $40 \mathrm{mg} / \mathrm{dl}$ su incidencia oscila entre el $2-19 \%$, en tanto que cuando el criterio diagnóstico es menos estricto $(<60 \mathrm{mg} / \mathrm{dl})$, su incidencia es del $30 \%{ }^{57,58}$.

El cuadro clínico traduce la disfunción energética neuronal secundaria a neuroglucopenia; en el ámbito de la $\mathrm{UCl}$, éste es variable, según los valores de glucemia y de las medidas terapéuticas previamente instauradas. Vriesendorp et $\mathrm{al}^{57}$ han establecido como factores de riesgo para la hipoglucemia: tratamiento con insulina, diabetes mellitus previa, sepsis grave, uso de vasopresores, detención no programada de la nutrición sin ajuste de la insulinoterapia, infusión de soluciones bicarbonatadas, insuficiencia renal y uso de técnicas de reemplazo renal continuo ${ }^{57}$.

Por su parte, Krinsley et $\mathrm{al}^{58}$ en una revisión retrospectiva analizaron los episodios de hipoglucemia de 102 pacientes críticos. El análisis de regresión logística multivariada reveló como factores de riesgo para la hipoglucemia grave: diabetes previa, shock séptico, insuficiencia renal, gravedad de la enfermedad crítica, puntuación del score APACHE II y la existencia de ventilación mecánica ${ }^{58}$. La mortalidad fue del 55,9\% entre los 102 pacientes que desarrollaron hipoglucemia grave, en tanto que en los 306 pacientes del grupo control la mortalidad fue del 39,5\% $(p=0,0057)^{58}$. Asimismo, se identificó a la hipoglucemia grave como un predictor independiente de mortalidad (OR: 2,28; IC 95\%: $1,41-3,70 ; p=0,008)$.

Mechanick et $\mathrm{al}^{59}$ en el año 2007 publicaron los resultados de su metaanálisis referente al riesgo relativo de hipoglucemia $(<40 \mathrm{mg} / \mathrm{dl})$ en los pacientes que recibían tratamiento intensivo con insulina. El análisis de los estudios incluidos (7 estudios, $n=3.728$ ) permite concluir que el riesgo de hipoglucemia es superior al $25 \%$ entre los pacientes a los que se les realizó tratamiento intensivo $(R R=4,97 ; \mathrm{IC}$ 95\%: 3,65-6,76; $\mathrm{p}<0,001)^{59}$.

Más recientemente, Arabi et $\mathrm{al}^{60}$ han establecido en 523 pacientes que la hipoglucemia ocurrió en el $16 \%$, es un factor de riesgo mayor el tratamiento intensivo con insulina (OR: 50,65; IC 95\%: 17,36-147,78; $\mathrm{p}<0001$ ). Por su parte, otras variables consideradas como factores de riesgo para hipoglucemia fueron sexo femenino, diabetes, ventilación mecánica, estancia prolongada en la $\mathrm{UCl}$ y técnicas de reemplazo renal continuo ${ }^{60}$.

El tratamiento de la hipoglucemia debe evitar la administración excesiva de glucosa parenteral, puesto que la sobrecorrección en las cifras de glucemia es potencialmente deletérea. En efecto, Suh et $\mathrm{al}^{61}$ en un modelo experimental en ratas confirmaron que la excesiva corrección con glucosa (período de reperfusión) y la generación de $\mathrm{RL}\left(\mathrm{O}_{2} \cdot{ }^{-}\right.$) vía activación de la NADPH (del inglés Nicotinamide Adenine Dinucleotide Phosphate) oxidasa neuronal con liberación masiva de cinc hacia el espacio extracelular provocan lesión y muerte neuronal, fenómenos que explican el daño neurológico persistente e irreversible ${ }^{61}$.

Los riesgos atribuibles a la hipoglucemia secundaria al tratamiento con insulina pueden evitarse o minimizarse con la implementación de algoritmos terapéuticos adecuados ${ }^{62}$, minimizar los errores de monitorización y evitar la sobrecorrección de la hipoglucemia con la finalidad de prevenir el daño neuronal secundario a la hipoglucemia "per se" y a la hiperglucemia de reperfusión ${ }^{61}$.

\section{Conclusiones}

La hiperglucemia de estrés y el control de la glucemia con insulina en el paciente crítico es un tema controvertido de la medicina intensiva. De acuerdo con el estado actual del conocimiento es posible establecer las siguientes conclusiones:

1. La hiperglucemia de estrés es un predictor pronóstico que actúa como un marcador de morbimortalidad durante la enfermedad crítica.

2. La infusión de insulina es capaz de revertir la resistencia periférica a ésta en el músculo esquelético mediante el aumento de la captación celular de glucosa y ejerce sus efectos protectivos mediante mecanismos directos (acción antiinflamatoria) e indirectos (control de la glucemia). 
3. La infusión de insulina debería iniciarse cuando la glucemia sea superior a 1,80 g/l; la vía intravenosa es de elección en el paciente crítico.

4. El nivel óptimo de glucemia parece ser de 1,40-1,80 g/l, en tanto que valores más estrechos de glucemia $(0,80$ $1,10 \mathrm{~g} / \mathrm{l})$ pueden ser aconsejados en pacientes quirúrgicos, en particular POCC.

5. En todo caso será necesario evitar la variabilidad de la glucemia puesto que se ha demostrado que ésta se comporta como un predictor independiente de mortalidad en la enfermedad crítica.

6. Es necesaria la realización e implementación de protocolos de control de la glucemia en cada $\mathrm{UCI}$, y es esencial la monitorización de la glucemia con el objetivo de minimizar el riesgo de hipoglucemia y optimizar el perfil glucémico.

7. La hipoglucemia (glucemia $<40 \mathrm{mg} / \mathrm{dl}$ ) es un predictor independiente de mortalidad. Su tratamiento debe evitar la administración excesiva de glucosa parenteral, puesto que la sobrecorrección en las cifras de glucemia es potencialmente deletérea (lesión neurológica irreversible).

\section{Conflicto de intereses}

Los autores declaran no tener ningún conflicto de intereses.

\section{Bibliografía}

1. Fahy BG, Sheehy AM, Coursin DB. Glucose control in the intensive care unit. Crit Care Med. 2009;31:1769-76.

2. Sakharova OV, Inzucchi SE. Endocrine assessments during critical illness. Crit Care Clin. 2007;23:467-90.

3. Collier B, Dossett LA, May AK, Díaz JJ. Glucose control and the inflammatory response. Nutr Clin Pract. 2008;23:3-15.

4. Langouche L, Vanhorebeek I, Van den Berghe G. Therapy insight: The effect of tight glycemic control in acute illness. Nat Clin Pract Endocrinol Metab. 2007;3:270-8.

5. Van den Berghe G, Wouters PJ, Bouillon R, Weekers F, Verwaest $C$, Schetz M, et al. Outcome benefit of intensive insulin therapy in the critically ill: Insulin dose versus glycemic control. Crit Care Med. 2003;31:359-66.

6. Corstjens AM, Van der Horst IC, Zijlstra JG, Groeneveld AB, Zijlstra F, Tulleken JE, et al. Hyperglycaemia in critically ill patients: Marker or mediator of mortality? Crit Care. 2006;10:216

7. Finney SJ, Zekveld C, Elia A, Evans TW. Glucose control and mortality in critically ill patients. JAMA. 2003;290:2041-7.

8. Gabbanelli V, Pantanetti S, Donati A, Principi T, Pelaia P. Correlation between hyperglycemia and mortality in a medical and surgical intensive care unit. Minerva Anestesiol. 2005;71: 717-725.

9. Kreutziger J, Wenzel V, Kurz A, Constantinescu MA. Admission blood glucose is an independent predictive factor for hospital mortality in polytraumatised patients. Intensive Care Med. 2009;35:1234-9.

10. Bochicchio GV, Sung J, Joshi M, Bochicchio K, Johnson SB, Meyer $\mathrm{W}$, et al. Persistent hyperglycemia is predictive of outcome in critically ill trauma patients. J Trauma. 2005;58:921-4.

11. Van den Berghe G, Wouters P, Weekers F, Verwaest C, Bruyninckx $F$, Schetz $M$, et al. Intensive insulin therapy in the critically ill patients. N Engl J Med. 2001;345:1359-67.
12. Krinsley JS. Effect of an intensive glucose management protocol on the mortality of critically ill adult patients. Mayo Clin Proc. 2004;79:992-1000.

13. Egi M, Bellomo R, Stachowski E, French CJ, Hart GK, Hegarty C, et al. Blood glucose concentration and outcome of critical illness: The impact of diabetes. Crit Care Med. 2008;36: 2249-55.

14. Pittas AG, Siegel RD, Lau J. Insulin therapy and in-hospital mortality in critically ill patients: A systematic review and meta-analysis of randomized controlled trials. JPEN J Parenter Enteral Nutr. 2006;30:164-72.

15. Van den Berghe G, Schoonheydt K, Becx P, Bruyninckx F, Wouters PJ. Insulin therapy protects the central and peripheral nervous system of intensive care patients. Neurology. 2005;64: 1348-1353.

16. Yendamuri S, Fulda GJ, Tinkoff GH. Admission hyperglycemia as a prognostic indicator in trauma. J Trauma. 2003;55:33-8.

17. Van den Berghe $G$. How does blood glucose control with insulin save lives in intensive care? J Clin Invest. 2004;114:1187-95.

18. Ellger B, Debaveye $Y$, Vanhorebeek I, Langouche L, Giulietti A, Van Etten E, et al. Survival benefits of intensive insulin therapy in critical illness: Impact of maintaining normoglycemia versus glycemia-independent actions of insulin. Diabetes. 2006;55: 1096-1105.

19. Van den Berghe G, Wilmer A, Hermans G, Meersseman W, Wouters PJ, Milants I, et al. Intensive insulin therapy in the medical ICU. N Engl J Med. 2006;354:449-61.

20. Devos P, Chiolero R, Van den Berghe G, Preiser JC. Glucose, insulin and myocardial ischemia. Curr Opin Clin Nutr Metab Care. 2006;9:131-9.

21. Rovilas A, Kotsou S. The influence of hyperglycemia on neurological outcome in patients with severe head injury. Neurosurgery. 2000;46:335-42.

22. Oddo M, Schmidt JM, Mayer S, Chioléro RL. Glucose control after severe brain injury. Curr Opin Clin Nutr Metab Care. 2008;11:134-9.

23. Jeschke MG, Klein D, Herndon DN. Insulin treatment improves the systemic inflammatory reaction to severe trauma. Ann Surg. 2004;239:553-60.

24. Yu WK, Li WQ, Li A, Li JS. Influence of acute hyperglycemia in human sepsis on inflammatory cytokine and contra-regulatory hormone concentrations. World J Gastroenterol. 2003;9: 1824-7.

25. Vanhorebeek I, Langouche L, Van den Berghe G. Glycemic and non glycemic effects of insulin: How do they contribute to a better outcome of critical illness. Curr Opin Crit Care. 2005;11: 304-311.

26. Mackenzie I, Ingle S, Zaidi S, Buczaski S. Tight glycaemic control: A survey of intensive care practice in large English hospitals. Intensive Care Med. 2005;31:1136.

27. Messoten D, Swinnwn JV, Vanderhoydonc F, Wouters PJ, Van den Berghe G. Contribution of circulating lipids to the improved outcome of critical illness by glycemic control with intensive insulin therapy. J Clin Endocrinol Metab. 2004;89:219-26.

28. Van den Berghe G, Wilmer A, Milants I, Wouters PJ, Bouckaert B, Bruyninckx $F$, et al. Intensive insulin therapy in mixed medical/ surgical intensive care units: Benefit versus harm. Diabetes. 2006;55:3151-9.

29. Reed CC, Stewart RM, Sherman M, Myers JG, Corneille MG, Larson $\mathrm{N}$, et al. Intensive insulin protocol improves glucose control and is associated with a reduction in intensive care unit mortality. $\mathrm{J}$ Am Surg Coll. 2007;204:1048-54.

30. Finfer S, Chittock DR, Su SY, Blair D, Foster D, Dhingra V, et al. Intensive versus conventional glucose control in critically ill patients. N Engl J Med. 2009;360:1283-97.

31. Brunkhorst FM, Engel C, Bloos F, Meier-Hellmann A, Ragaller M, Weiler $\mathrm{N}$, et al. Intensive insulin therapy and pentastarch resuscitation in severe sepsis. N Engl J Med. 2008;358:125-39. 
32. Preiser JC, Devos P, Ruiz-Santana S, Mélot C, Annane D, Groeneveld J, et al. A prospective randomised multi-centre controlled trial on tight glucose control by intensive insulin therapy in adult intensive care units: The GluControl study. Intensive Care Med. 2009;35:1738-48.

33. Arabi YM, Dabbagh OC, Tamim HM, Al-Shimemeri AA, Memish $\mathrm{ZA}$, Haddad $\mathrm{SH}$, et al. Intensive versus conventional insulin therapy: A randomized controlled trial in medical and surgical critically ill patients. Crit Care Med. 2008;36:3190-7.

34. Dellinger RP, Levy MM, Carlet JM, Bion J, Parker MM, Jaeschke $\mathrm{R}$, et al. Surviving sepsis campaign: International guidelines for management of severe sepsis and septic shock: 2008. Crit Care Med. 2008;36:296-327.

35. Krinsley JS, Preiser JC. Moving beyond tight glucose control to safe effective glucose control. Crit Care. 2008;12:149.

36. Griesdale DEG, De Souza RJ, Van Dam RM, Heyland DK, Cook DJ, Malhotra A, et al. Intensive insulin therapy and mortality among critically ill patients: A meta-analysis including NICE-SUGAR study data. CMAJ. 2009;180:821-7.

37. Moghissi ES, Korytkowski MT, DiNardo M, Einhorn D, Hellman R, Hirsch IB, et al. American Association of Clinical Endocrinologists and American Diabetes Association consensus statement on inpatient glycemic control. Diabetes Care. 2009;32:1119-31.

38. Ali NA, O’Brien JM, Dungan K, Phillips G, Marsh CB, Lemeshow S, et al. Glucose variability and mortality in patients with sepsis. Crit Care Med. 2008;36:2316-21.

39. Egi M, Bellomo R, Stachowski E, French CJ, Hart G. Variability of blood glucose concentration and short-term mortality in critically ill patients. Anesthesiology. 2006;105:244-52.

40. Krinsley JS. Glycemic variability: A strong independent predictor of mortality in critically ill patients. Crit Care Med. 2008;36:3008-13.

41. Dossett LA, Cao H, Mowery NT, Dortch MJ, Morris Jr JM, May AK. Blood glucose variability is associated with mortality in the surgical intensive care unit. Am Surg. 2008;74:679-85.

42. Ali NA, Krinsley JS, Preiser JC. Glucose variability in critically ill patients. En: Vincent JL, editor. 2009 Yearbook in Intensive Care and Emergency Medicine. Berlin: Springer-Verlag; 2009. p. 728-37.

43. Malmberg K, Ryden L, Hamsten A, Herlitz J, Waldenström A, Wedel $\mathrm{H}$. Effects of insulin treatment on cause-specific one year mortality and morbidity in diabetic patients with acute myocardial infarction: DIGAMI Study Group: Diabetes insulineglucose in acute myocardial infarction. Eur Heart J. 1996;17: 1337-1344.

44. Malmberg K, Ryden L, Wedel H, Birkeland K, Bootsma A, Dickstein $\mathrm{K}$, et al. Intense metabolic control by means of insulin in patients with diabetes mellitus and acute myocardial infarction (DIGAMI 2): Effects on mortality and morbidity. Eur Heart J. 2005;26:650-61.

45. Mehta SR, Yusuf S, Díaz R, Zhu J, Pais P, Xavier D, et al. Effect of glucose-insulin-potassium infusion on mortality in patients with acute ST-segment elevation myocardial infarction: the CREATE-ECLA randomized controlled trial. JAMA. 2005;293: 437-446.

46. Ouattara A, Lecomte P, Le Manach Y, Landi M, Jacqueminet S, Platonov $\mathrm{I}$, et al. Poor intraoperative blood glucose control is associated with a worsened hospital outcome after cardiac surgery in diabetic patients. Anesthesiology. 2005;103: 687-94.
47. Zimmerman CR, Mlynarek ME, Jordan JA, Rajda CA, Horst HM. An insulin infusion protocol in critically ill cardiothoracic surgery patients. Ann Pharmacother. 2004;38:1123-9.

48. Gandhi GY, Nuttall GA, Abel MD, Mullany CJ, Schaff HV, O’Brien $\mathrm{PC}$, et al. Intensive intraoperative insulin therapy versus conventional glucose management during cardiac surgery. A randomized trial. Ann Intern Med. 2007;146:233-43.

49. Zerr KJ, Furnary AP, Grunkemeier GL. Glucose control lowers the risk of wound infection in diabetics after open-heart operations. Ann Thorac Surg. 1997;63:356-61.

50. Furnary AP, Zerr KJ, Grunkemeier GL. Continuous intravenous insulin infusion reduces the incidence of deep sternal wound infection in diabetic patients after cardiac surgical procedures. Ann Thorac Surg. 1999;67:352-62.

51. Van Cromphaut SJ, Vanhorebeek I, Van den Berghe G. Hyperglycaemia and neurological injury. Curr Opin Anaesthesiol. 2008;21:565-9.

52. Baird TA, Parsons MW, Planh T, Butcher KS, Desmond PM, Tress $\mathrm{BM}$, et al. Persistent poststoke hyperglycemia is independently associated with infarct expansion and worse clinical outcome. Stroke. 2003;34:2208-14.

53. Frontera JA, Fernández A, Claassen J, Schmidt M, Schumacher HC, Wartenberg K, et al. Hyperglycemia after SAH: Predictors, associated complications, and impact on outcome. Stroke. 2006;37:199-203.

54. Bilotta F, Spinelli A, Giovannini F, Doronzio A, Delfini R, Rosa G. The effect of intensive insulin therapy on infection rate, vasospasm, neurologic outcome and mortality in neurointensive care unit after intracranial aneurysm clipping in patients with acute subarachnoid haemorrhage: A randomized prospective pilot trial. J Neurosurg Anesthesiol. 2007;19:156-60.

55. Vespa P, Boonyaputthikul R, McArthur DL, Miller C, Etchepare M, Bergsneider $M$, et al. Intensive insulin therapy reduces microdialysis glucose values without altering glucose utilization or improving the lactate/pyruvate ratio after traumatic brain injury. Crit Care Med. 2006;34:850-6.

56. Devos P, Preiser JC. Current controversies around tight glucose control in critically ill patients. Curr Opin Clin Nutr Metab Care. 2007;10:206-9.

57. Vriesendorp TM, Van Santen S, DeVries JH, De Jonge $E$, Rosendaal FR, Schultz MJ, et al. Predisposing factors for hypoglycemia in the intensive care unit. Crit Care Med. 2006;34: 96-101.

58. Krinsley JS, Grover A. Severe hypoglycemia in critically ill patients: Risk factors and outcomes. Crit Care Med. 2007; 35:2262-7.

59. Mechanick JI, Handelsman Y, Bloomgarden ZT. Hypoglicemia in the intensive care unit. Curr Opin Clin Nutr Metab Care. 2007;10:193-6.

60. Arabi YM, Tamim HM, Rishu AH. Hypoglycemia with intensive insulin therapy in critically ill patients: Predisposing factors and association with mortality. Crit Care Med. 2009;37:2536-44.

61. Suh SW, Gum ET, Hamby AM, Chan PH, Swanson RA. Hypoglycemic neuronal death is triggered by glucose reperfusion and activation of neuronal NADPH oxidase. J Clin Invest. 2007; 117:910-8.

62. Nasraway Jr SA. Sitting on the horns of a dilemma: Avoiding severe hypoglycemia while practicing tight glycemic control. Crit Care Med. 2007;35:2435-7. 